\title{
Contribution de la Diversification des Exportations a la Croissance Economique en Côte d'Ivoire
}

\author{
Kouassi Dèdjé Sylvestre Eric,
} Université Péléforo Gon Coulibaly de Korhogo, Côte d'Ivoire

Doi:10.19044/esj.2020.v16n10p297 URL:http://dx.doi.org/10.19044/esj.2020.v16n10p297

\section{Résumé}

Cette étude examine la contribution de la diversification des exportations à la croissance économique en Côte d'Ivoire. Pour réaliser l'étude l'article estime une fonction de production de Cobb-Douglas augmentée. La méthode d'estimation utilisée est celle des moindres carrés généralisés avec correction des problèmes d'autocorrélation et d'hétéroscédasticité avec la méthode de Cochrane-Orcutt avec une matrice variance-covariance robuste. Les résultats montrent que le processus de diversification des exportations à la marge intensive stimule la croissance économique à travers notamment le contrôle du stock de capital physique et le facteur travail. Ainsi, l'étude recommande dans un premier temps la réorganisation des secteurs traditionnels exportateurs et dans un deuxième temps l'extension de la transformation à la gamme supérieure de produits exportés.

Mots clés : Diversification des exportations, croissance économique, Moindre carrés généralisés (MCG) 


\title{
Contribution of Exports Diversification to Economic Growth in Côte d'Ivoire
}

\author{
Kouassi Dèdjé Sylvestre Eric, \\ Université Péléforo Gon Coulibaly de Korhogo, Côte d'Ivoire
}

\begin{abstract}
This paper focuses on examining the contribution of export diversification to economic growth in Côte d'Ivoire. To carry out the study, the article estimates an increased Cobb-Douglas production function. The estimation method used is that of generalized least squares with correction of autocorrelation and heteroskedasticity problems. This is together with the Cochrane-Orcutt method with a robust variance-covariance matrix. The results show that the process of diversification of exports at the intensive margin stimulates economic growth through notably the control of the stock of physical capital and the labor factor. Firstly, the study recommends the reorganization of traditional export sectors and secondly the extension of processing to the higher range of exported products.
\end{abstract}

Keywords: Export Diversification, Economic Growth, Generalized Least Squares (GLS)

\section{Introduction}

La Côte d'Ivoire a connu deux grandes périodes de forte croissance économique dans son histoire. La première période qui part de 1960 à 1970 a été qualifiée de miracle ivoirien avec un taux de croissance économique moyen annuel de $8 \%$. Toutefois, cette croissance s'est estompée progressivement pour diverses raisons, dont la forte concentration des exportations sur les produits de rente et la série de chutes des prix de ces produits au cours du temps.

La deuxième période (1990-2016) est marquée par les petites reprises suite au plan d'ajustement dont les effets ont été limités par les crises. La croissance économique fut alors limitée jusqu'en 2012 où l'économie ivoirienne a enregistré une reprise. Le rythme moyen de la croissance économique est supérieur à $8 \%$, couplé à une inflation faible (Ehrhart, 2015). Selon les estimations de l'Agence Française de Développement (AFD), le taux de croissance observé en $2012(10,7 \%)$ a permis d'atteindre le niveau de Produit Intérieur Brut (PIB) qui aurait été obtenu en l'absence de récession en 
2011 et si la tendance de croissance des cinq années précédentes s'était poursuivie. Toutefois, le niveau de PIB simulé qui aurait été atteint en 2014 si, depuis 2000, le pays avait eu chaque année le taux de croissance moyen de l'Afrique Subsaharienne (ASS) est resté substantiellement supérieur au niveau effectivement réalisé. Cela prouve qu'une forte croissance économique soutenue sur plusieurs années est encore nécessaire pour combler ce retard (Ehrhart, 2015).

La consolidation d'une forte croissance économique va en partie dépendre de la capacité du pays à stimuler le secteur privé et à gérer les risques internes et externes (Morisset, 2012). Pour maîtriser ces risques et générer une croissance durable et plus inclusive, l'économie ivoirienne pourrait poursuivre ses efforts de diversification et augmenter la valeur ajoutée de ses exportations en s'inspirant des pays émergents qui fabriquent des produits de plus en plus sophistiqués.

Cette dynamique peut se traduire par le passage vers une structure de production plus variée, avec le lancement de nouveaux produits ou le développement de produits préexistants, notamment des produits de meilleure qualité (fonds monétaire international, 2014). La première approche correspond au développement de la marge extensive ou horizontale et la deuxième correspond à celui de la marge intensive ou diversification verticale (Cottet et al., 2012; Taylor, 2007). Cela nécessite une transformation structurelle qui assure un transfert de ressources du secteur primaire au secteur secondaire (Banque Mondiale, 2015).

Pourtant, en Côte d'Ivoire, ce processus semble inachevé en dépit des efforts déployés au lendemain de l'indépendance. En effet, le modèle ivoirien de croissance reste encore tributaire des produits agricoles, même s'il s'oriente vers davantage de diversification grâce à l'amélioration du climat des affaires. Bien qu'en constant recul depuis les années 1980 et ce au profit du secteur de l'industrie (27\% du PIB), le secteur agricole ( $24 \%$ du PIB) reste le premier pourvoyeur d'emplois (avec $45 \%$ des emplois), suivi du secteur du commerce et des services (avec $44 \%$ des emplois). Le secteur industriel qui dispose d'un potentiel important de valeurs ajoutées n'occupe que $12,5 \%$ des travailleurs. Cette configuration de la croissance économique en Côte d'Ivoire limite la capacité de création rapide et de répartition des richesses.

L'expérience et la théorie économique ont montré que la diversification est utile tant pour accroitre la résilience d'une économie que pour augmenter sa productivité au cours du temps (Banque Mondiale, 2017 ; Chatri et al., 2018). Une plus grande diversification permet de créer des effets multiplicateurs ou des effets de diffusion (effets spillovers) ainsi qu'une hausse de la productivité (Melitz, 2003). Au-delà de la gestion des risques, la diversification devient un instrument pour améliorer la qualité de la croissance en stimulant l'innovation technologique et la productivité de la main-d'œuvre 
(Romer, 1990). La diversification peut également contribuer à la croissance en augmentant le nombre de secteurs et par conséquent d'opportunités d'investissement et en réduisant le risque des investisseurs (Acemoglu \& Zilibotti, 1997).

De tout ce qui précède, l'on est en droit de s'interroger, dans le contexte actuel ivoirien et à quelles conditions, la poursuite de la diversification est-elle susceptible de stimuler durablement le processus de création des richesses?

Afin de répondre à cette question, cette étude examine la contribution de la diversification des exportations à la croissance économique en Côte d'Ivoire. Pour ce faire, elle a recours à un modèle économétrique pour estimer les effets de long terme d'un accroissement de la diversification des exportations sur les variations du PIB réel.

La section suivante procède à une recension des écrits pertinents. La section 3 présente la méthodologie de l'étude. Les résultats économétriques sont analysés dans la section 4 . La section 5 conclut l'étude.

\section{Revue de la littérature}

Le débat sur la diversification et son rôle dans les dynamiques de croissance ne s'est pas forcément accompagné d'un consensus. La théorie traditionnelle du commerce international et certains résultats des nouvelles théories accréditent la thèse d'une relation croissante entre la spécialisation du commerce international et croissance (Ben Hammouda et al., 2009). Cependant, des auteurs comme Ben Hammouda et al. (2009) relativisent en montrant que les effets de l'échange sur la croissance à long terme dépendent de la nature de la spécialisation sectorielle (Ben Hammouda et al., 2009). Ainsi, certaines spécialisations seraient plus favorables à la croissance que d'autres. Ce point de vue atteste l'hypothèse de Prebisch (1959) selon laquelle, sur une longue période, le prix des biens non transformés pris dans leur ensemble déclinerait relativement à celui des biens manufacturés, appauvrissant les pays qui les exportent. Cela fait de l'industrialisation d'une économie un enjeu majeur de l'élargissement de la base exportatrice.

Plusieurs études théoriques et empiriques établissent que la diversification pourrait avoir un effet positif sur la croissance. Par exemple, le modèle de Romer (1990) a introduit un effet bénéfique de la diversification exprimé par la disponibilité des intrants au sein d'une économie, pouvant contribuer à l'accroissement de la productivité du travail et du capital humain. Acemoglu et Zilibotti (1997) montrent que la diversification peut également contribuer à la croissance en augmentant le nombre de secteurs et par conséquent d'opportunités d'investissement et en réduisant le risque des investisseurs. 
Les études qui éclaircissent le concept de diversification des exportations et son rôle dans la dynamique de croissance ne manquent pas. Cottet et al. (2012) présentent quelques-unes qui ont décomposé la croissance des exportations selon deux directions (Klinger \& Lederman, 2011 ; Cadot et al., 2011 ; Hummels \& Klenow, 2005) : il ressort que cette croissance peut provenir d'une part de l'augmentation des nouveaux produits exportés ou la marge extensive et d'autre part de l'accroissement d'exportations de produits déjà existantes, appelée la marge intensive. Lorsque la marge extensive domine la marge intensive, l'apparition de nouveaux produits d'exportations peut être un moteur de croissance économique. Cela est vrai si l'apparition de nouveau produit provient d'un processus de remontée de la chaîne de la valeur ajoutée ou d'un processus d'innovation faisant l'objet d'une déposition de brevet ou d'une simple imitation (Klinger \& Lederman, 2011 ; Cadot et al., 2011 ; Hummels \& Klenow, 2005 ; Pham \& Martin, 2007).

Greenaway, Morgan et Wright (1999) ont analysé l'influence de la dynamique de la croissance des exportations sur la croissance, en explorant deux pistes. Premièrement, ils examinent le lien exportations-croissance de manière dynamique, en proposant une approche plus rigoureuse que celle qui avait été tentée auparavant. Deuxièmement, ils explorent le rôle de la composition des exportations dans la détermination de la performance de croissance. A partir des données compilées sur un panel de 69 pays et d'un modèle dynamique, ces auteurs démontrent l'existence d'une forte relation positive entre les exportations et la croissance. En outre, il est évident que la composition de ces exportations est importante pour déterminer la vigueur de la croissance. À ce propos, Herzer et Nowak-Lehnmann (2006) examinent l'hypothèse d'une croissance tirée par les exportations à l'aide de données chronologiques annuelles du Chili dans le cadre d'une fonction de production. Il aborde les limites de la littérature existante et met l'accent sur l'impact des exportations de produits manufacturés et primaires sur la croissance de la productivité. Afin d'identifier l'existence et le sens de l'influence des exportations de produits manufacturés et de produits primaires sur la croissance économique par le biais d'une augmentation de la productivité, ils utilisent plusieurs techniques de cointégrations, à équation unique et en systèmes. Les résultats des estimations peuvent être interprétés comme la preuve que les exportations de produits manufacturés boostent la productivité tandis que les exportations primaires ont un effet restrictif sur la productivité.

Dans le même ordre d'idée Anwesha, A. et Acharyya, R. (2013) examine la relation exportations-croissance à des niveaux désagrégés (désagrégation au niveau des pays et au niveau des exportations) en se concentrant sur la diversification et la composition des exportations des pays. A partir d'un échantillon de 65 pays sur la période 1965-2005, l'estimation par panel dynamique révèle que la diversification et la composition des 
exportations sont des déterminants importants de la croissance économique après la prise en compte des incidences d'autres variables telles que le revenu retardé, l'investissement et les infrastructures. Il montre, par ailleurs, qu'il existe un niveau critique de concentration des exportations au-delà duquel la spécialisation croissante des exportations entraîne une croissance plus forte. En dessous de ce niveau critique, la diversification des exportations renforce la croissance du PIB. La croissance des exportations de haute technologie contribue également à stimuler la production. La relation se renforce davantage pour les pays dont la part des exportations manufacturières dans leurs exportations totales est supérieure à la moyenne mondiale. Les auteurs affirment que ces résultats sont robustes, même lorsque l'ensemble de données est classé dans quatre sous-groupes en fonction de la relation croissanceexportation.

\section{Méthodologie de l'étude Modèle}

Cette étude s'inspire des travaux de Hakim et al. (2010) effectués sur la diversification des exportations en Afrique et des travaux des modèles de croissance endogène, notamment le modèle du « learning by doing » d'Arrow (1962). Elle tente également de prendre en compte l'hypothèse de la « diffusion de la connaissance » de Romer (1986). Ces auteurs mettent en avant le rôle de ces facteurs. Dans ces conditions, «l'apprentissage par la pratique » et «la diffusion de la connaissance » peuvent être captées à travers l'expansion $\mathrm{du}$ commerce et la diversification des exportations pour stimuler une croissance durable. En effet, la poursuite de la diversification des exportations induit des changements susceptibles d'affecter le paramètre de la productivité totale des facteurs.

Le modèle utilisé dans cette étude découle de la vision néoclassique de la fonction de production (Solow, 1957). Selon cette vision, la croissance découle de l'accumulation des facteurs de production et l'amélioration de la productivité totale des facteurs. Le point de départ de cette modélisation est la fonction de production de type Cobb-Douglas suivante :

$$
Y_{t}=A_{t} K_{t}^{\beta_{1}} L_{t}^{\beta_{2}}
$$

où les paramètres à estimer $\beta_{1}$ et $\beta_{2}$ représentent les élasticités de la production respectivement par rapport au stock de capital et au travail. $Y_{t}, K_{t}$ et $L_{t}$ désignent successivement la production, le stock de capital et le facteur travail du pays i à la période t. $A_{t}$ représente la productivité totale des facteurs, qui reflète le niveau de la technologie et d'efficacité économique. En adoptant une spécification proche de celle de Keho (2012), la productivité totale des facteurs peut s'écrire de la manière suivante :

$$
A_{t}=A_{0} e^{\left(\beta_{3} D_{t}+\varepsilon_{t}\right)}
$$


où $A_{0}$ est une constante $; D_{t}$ est un indicateur de diversification des exportations et $\varepsilon_{\mathrm{t}}$ représente les termes d'erreurs indépendamment et identiquement distribués (i.i.d). En combinant les équations (1) et (2) et la linéarisant par la transformation logarithmique, on obtient :

$$
\ln Y_{t}=\ln A_{t}+\beta_{1} \ln K_{t}+\beta_{2} \ln L_{t}+\ln A_{0}+\beta_{3} D_{t}+\varepsilon_{t}
$$

En posant $y_{t}=\ln Y_{t}, k_{t}=\ln K_{t}, l_{t}=\ln L_{t}$, et $\beta_{0}=\ln A_{0}$ on obtient l'équation empirique finale à estimer suivante :

$$
y_{t}=\beta_{0}+\beta_{1} k_{t}+\beta_{2} l_{t}+\beta_{3} D_{t}+\varepsilon_{t}
$$

où $y_{t}$ désigne le logarithme de la production en volume, $k_{t}$ le logarithme du stock de capital physique, $l_{t}$ le logarithme de la main-d'œuvre et $\beta_{0}$ est une constante qui égale au logarithme de $A_{0}$.

\section{Variables et sources de données}

L'équation 4 est estimée à partir de séries temporelles concernant la Côte d'Ivoire au cours de la période 1962-2010.

Les données relatives aux trois premières variables du modèle proviennent de Penn World Table 9.0 (PWT9.0). Il s'agit notamment du logarithme du PIB réel en millions de dollars de $2011\left(y_{t}\right)$, du logarithme du stock de capital physique en millions de dollars à prix constant de 2011 ( $\left.k_{t}\right)$ et $\mathrm{du}$ logarithme de la main-d'œuvre correspondant au nombre de personnes engagées en millions $\left(l_{t}\right)$. Le capital et le travail étant reconnus dans la théorie économique comme étant des facteurs explicatifs majeurs de la croissance économique, les signes de leur coefficient sont supposés être positifs.

La diversification des exportations est mesurée par l'indice de concentration de Theil $\left(D_{t}\right)$. Lorsque cet indice augmente, la diversification des exportations diminue et vice-versa. Un signe négatif du coefficient associé à cette variable signifie alors un impact positif de la diversification des exportations sur la croissance. Un intérêt à utiliser cette mesure est qu'elle est décomposable en marge extensive et en marge intensive. Cela permet ainsi d'estimer l'impact des deux directions de la diversification des exportations sur la croissance économique en Côte d'Ivoire. Les données sur les indices de concentration de Theil proviennent de la base de données sur les indicateurs de diversification et de la qualité des exportations du Fonds Monétaire International (FMI) disponible, sur la période 1962-2010.

Dans le modèle utilisé, deux variables muettes sont introduites pour analyser l'effet des deux grandes crises qu'a connues la Côte d'Ivoire. La première crise, qui court de 1980 à 1990, est cernée par une variable dichotomique qui prend la valeur un entre 1980 et 1990 et la valeur zéro partout ailleurs. La seconde est liée à la crise militaire qui a débuté avec le coup d'État de 1999 pour s'achever en 2010. Elle est captée par une variable 
dichotomique qui prend la valeur 1 sur la période 1999-2010 et la valeur 0 endehors de cette période.

\section{Résultats}

L'étude de la stationnarité est un préalable à l'étude des séries temporelles en économie. En effet, lorsque des séries sont non-stationnaires, elles n'ont aucune force de rappel lorsqu'elle s'éloigne de sa moyenne sous l'effet de chocs. Par conséquent, l'étude de la relation entre des séries non stationnaires à partir des techniques d'estimation ordinaire peut conduire à des régressions fallacieuses. Dans le cas de la Côte d'Ivoire, plusieurs chocs, économiques et non-économiques, sur la période d'étude rendent nécessaire l'application d'un test de stationnarité sur les séries. Il s'agit de déterminer le degré d'intégration de chacune des séries prise individuellement. Le test le plus utilisé est le test Dickey-Fuller Augmenté (ADF) dont l'hypothèse nulle est « la série a une racine unitaire ». Ce dernier permet de tester la stationnarité d'une série en niveau, en différences premières, en différences secondes et plus, sous l'hypothèse nulle « la série a une racine unitaire ». Les résultats du test de Dickey-Fuller augmenté (ADF) avec dérive dans le Tableau 1 à la page 9 (suivante) montre qu'elles sont stationnaires en niveau, c'est-à-dire qu'elles sont toutes intégrées d'ordre zéro.

Tableau 1. Test de Dickey-Fuller Augmenté (ADF)

\begin{tabular}{llcccr}
\hline \hline Variables & Statistiques & Sans constante & Tendance & Avec Dérive & Décision \\
\hline \multirow{2}{*}{$\boldsymbol{y}_{\boldsymbol{t}}$} & $Z(t)$ & 4,511 & $-3,119$ & $-4,496$ & Stationnaire \\
& P-value & & 0,1019 & 0,000 & \\
\hline \multirow{2}{*}{$\boldsymbol{k}_{\boldsymbol{t}}$} & $Z(t)$ & 5,363 & $-0,860$ & $-5,570$ & Stationnaire \\
& P-value & & 0,9603 & 0,000 & \\
\hline \multirow{2}{*}{$\boldsymbol{l}_{\boldsymbol{t}}$} & $Z(t)$ & 10,842 & 2,327 & $-7,301$ & Stationnaire \\
& P-value & & 1,000 & 0,000 & \\
\multirow{2}{*}{$\boldsymbol{D}_{\boldsymbol{t}}$} & $Z(t)$ & $-0,540$ & $-3,387$ & $-2,810$ & Stationnaire \\
& P-value & & 0,0531 & 0,0036 & \\
\hline \hline
\end{tabular}

Source : calculs de l'auteur

La méthode des moindres carrés ordinaires reste valable à condition que les résidus respectent certaines propriétés telles que la normalité, l'homoscédasticité et l'absence d'autocorrélation des résidus. Pour l'étude de la normalité des résidus, le test de Jarque-Bera, dont l'hypothèse nulle $\left(\mathrm{H}_{0}\right)$ est la normalité des résidus, a été effectué. Les résultats de ce test indiquent que les résidus sont normalement distribués (Tableau 2). Il y a 99,84\% de chance de rejeter $\mathrm{H}_{0}$ à tort. Le test de Breusch-Pagan/Cook-Weisberg, dont l'hypothèse nulle $\left(\mathrm{H}_{0}\right)$ est que la variance est constante, permet d'étudier l'hétéroscédasticité des séries utilisées. Ce test confirme l'existence de l'hétéroscédasticité en montrant que l'on a $0,14 \%$ de chance rejeter à tort $\mathrm{H}_{0}$. Le test de Breusch-Godfrey d'autocorrélation avec pour l'hypothèse nulle $\left(\mathrm{H}_{0}\right)$ 
absence d'autocorrélation indique qu'il n'y a aucune chance (0\%) d'accepter $\mathrm{H}_{0}$. Les résidus sont auto-corrélés d'ordre un.

Tableau 2. Test sur les résidus

\begin{tabular}{|c|c|c|}
\hline Tests & Statistiques & Probabilité \\
\hline $\begin{array}{l}\text { Test de Normalité Skewness/Kurtosis } \\
\mathrm{H}_{0}: \text { normalité }\end{array}$ & $\operatorname{adj} \operatorname{chi} 2(2)=0$ & 0,9984 \\
\hline $\begin{array}{l}\text { Test d'hétéroscédasticité de Breush-Pagan/Cook- } \\
\text { Weisberg } \\
\mathrm{H}_{0} \text { : Variance constante }\end{array}$ & Chi2(1) $=10,17$ & 0,0014 \\
\hline $\begin{array}{l}\text { Test d'autocorrélation sérielle de Breusch-Godfrey } \\
\mathrm{H}_{0}: \text { Absence d'autocorrélation d'ordre } 1\end{array}$ & $\begin{array}{l}\text { Chi2(1)= } \\
34,014\end{array}$ & 0.0000 \\
\hline
\end{tabular}

Source : l'auteur, à partir du logiciel Stata 14.0

$\mathrm{Au}$ vu des résultats susmentionnés, on a recours à la méthode des moindres carrés généralisés tout en corrigeant l'autocorrélation par la méthode Cochrane-Orcutt et l'hétéroscédasticité à travers la production d'une matrice variance-covariance robuste.

Les résultats obtenus sont présentés dans la colonne (1) du Tableau 3. Le modèle estimé est globalement significatif au seuil de $1 \%$ et le $\mathrm{R}^{2}$ indique que $59 \%$ des variations du PIB sont expliquées par les variables du modèle.

Tableau 3. Résultats des estimations des déterminants de $y_{t}$

\begin{tabular}{|c|c|c|c|c|}
\hline & $\begin{array}{l}1) \\
1962-2010 \\
\end{array}$ & $\begin{array}{l}2) \\
1962-1986 \\
\end{array}$ & $\begin{array}{l}3) \\
1987-2010 \\
\end{array}$ & $\begin{array}{l}4) \\
1987-2010 \\
\end{array}$ \\
\hline $\mathrm{kt}$ & $\begin{array}{l}0.42 * * \\
(0.02)\end{array}$ & $\begin{array}{c}0.75^{* * * *} \\
(0.00)\end{array}$ & $\begin{array}{c}2.10^{* * *} \\
(0.00)\end{array}$ & $\begin{array}{c}2.09^{* * * *} \\
(0.00)\end{array}$ \\
\hline lt & $\begin{array}{c}0.37 * * * \\
(0.00)\end{array}$ & $\begin{array}{l}-0.12 \\
(0.79)\end{array}$ & $\begin{array}{c}0.59 * * * \\
(0.00)\end{array}$ & $\begin{array}{c}0.61 * * * \\
(0.00)\end{array}$ \\
\hline Dt & $\begin{array}{c}0.02 \\
(0.69)\end{array}$ & $\begin{array}{c}0.07 \\
(0.24)\end{array}$ & $\begin{array}{c}-0.09 * * * \\
(0.00)\end{array}$ & \\
\hline DIt & & & & $\begin{array}{c}-0.09 * * * \\
(0.00)\end{array}$ \\
\hline DEt & & & & $(0.14)^{-0.08}$ \\
\hline crise1999 & $\begin{array}{l}0.01 \\
(0.45)\end{array}$ & & $\begin{array}{l}-0.03 \\
(0.26)\end{array}$ & $\begin{array}{l}-0.03 \\
(0.24)\end{array}$ \\
\hline crise 1980 & $\begin{array}{l}-0.08 \\
(0.20)\end{array}$ & $\begin{array}{l}-0.16 * * * \\
(0.00)\end{array}$ & & \\
\hline $\mathrm{C}$ & $\begin{array}{l}4.95 * * \\
(0.02)\end{array}$ & $\begin{array}{l}1.53 \\
(0.52) \\
\end{array}$ & $\begin{array}{l}-15.00 * * \\
(0.02)\end{array}$ & $\begin{array}{l}-14.93 * * \\
(0.02)\end{array}$ \\
\hline $\mathrm{N}$ & 48 & 25 & 22 & 22 \\
\hline $\mathrm{R} 2$ & 0.688 & 0.639 & 0.968 & 0.971 \\
\hline adj. R2 & 0.651 & 0.566 & 0.960 & 0.961 \\
\hline
\end{tabular}

Note - $p$-values in parenthèses and ${ }^{*} p<0.10,{ }^{* *} p<0.05,{ }^{* * *} p<0.01$

Source : calculs de l'auteur. 
En ce qui concerne la significativité individuelle des coefficients des variables du modèle, seuls les coefficients associés au stock de capital et à l'emploi sont significatifs. La diversification n'a donc pas d'effet significatif sur la croissance économique.

La stabilité du modèle estimé au cours du temps est également analysée en utilisant les tests de cusum et de cusum au carré (voir Figure 1).

Figure 1. Test de Cusum pour la période 1962-2010

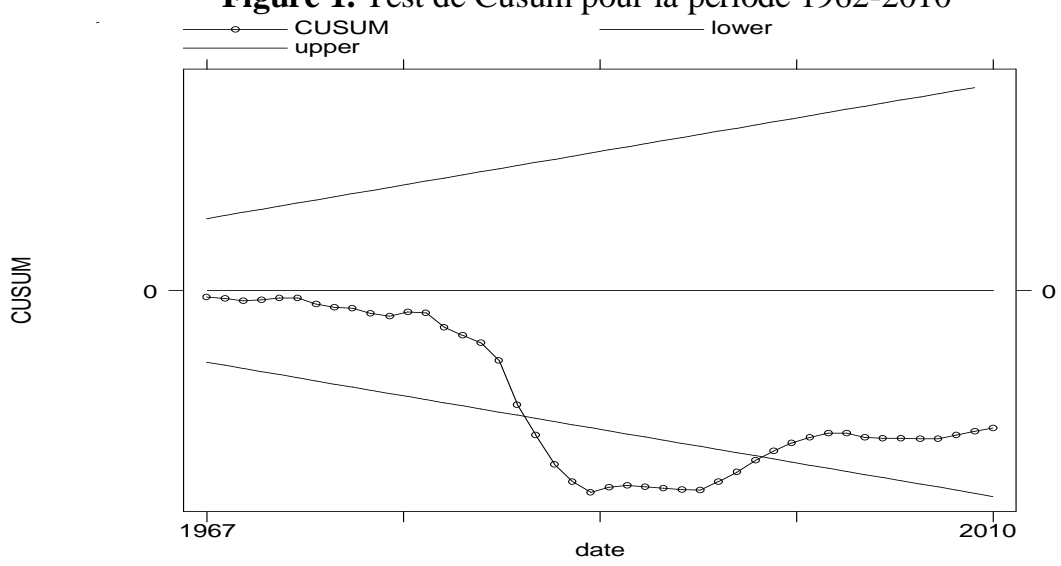

Source : l'auteur

Ces tests se présentent sous forme graphique et la valeur de la statistique doit alors évoluer, sous l'hypothèse nulle de stabilité de la relation, entre deux droites représentant les bornes de l'intervalle. Ce test de cusum est donc réalisé pour tester l'hypothèse de stabilité des relations de long terme estimées relation entre le PIB et les variables explicatives. Ainsi, les coefficients sont dits stables au cours du temps lorsque les résidus doivent rester dans les intervalles définis. Les résultats de ce test montrent qu'il y a une instabilité autour de la $26^{\text {ème }}$ période.

Cette instabilité peut être le résultat d'une rupture structurelle ou «structural break» qu'il convient de tester. On utilise le test de rupture structurelle avec une date connue, qui utilise un test de Wald ou de LikelihoodRatio. L'hypothèse nulle de ce test est qu'il n'y a pas de rupture structurelle. Les résultats confirment cette hypothèse à partir de la $27^{\text {ème }}$ période qui correspond à l'année 1988. Cela se justifie par les plans de sauvetage mis en place depuis le début des années 80 et les programmes d'ajustements structurels des années 90 (voir Tableau 4).

Tableau 4. Test de wald de rupture de structure étant donnée les dates

\begin{tabular}{lcc}
\hline \hline & Année 1987 & Année 1988 \\
\hline Hypothèse nulle $\mathrm{H}_{0}$ & Pas de rupture structurelle \\
Chi2 (4) & 18,22 & 7,34 \\
Probabilité & 0,0011 & 0,1188 \\
\hline \hline
\end{tabular}

Source : l'auteur à partir du logiciel Stata 
La colonne (2) du Tabelau 3 présente les résultats des régressions sur le période 1962-1987. D'après ces résultats, seul le stock de capital et la crise des années 80 ont eu un impact significatif sur la croissance. En effet, les coefficients associés au stock de capital et à la variable crise 1980 sont significativement différents de zéro. Ainsi, ni la main-d'œuvre ni la diversification des exportations n'a eu d'impact significatif sur la croissance économique. Cela pourrait s'expliquer par le fait que la croissance à cette époque pourrait être le résultat d'un effet d'opportunité et non d'une véritable stratégie de diversification. Par ailleurs, la crise de 1980 a limité la dynamique de croissance en Côte d'Ivoire. La colonne (3) du Tableau 3 montre les résultats obtenus sur la période 1987-2010. Afin de prendre en compte ce changement structurel dans la dynamique de croissance en Côte d'Ivoire, on se propose d'estimer l'équation de base pour les deux sous-périodes 19621986 et 1987-2010. Les résultats sont stables, au regard du test de cusum au carré pour cette période (voir Figure 2).

Figure 2. Test de Cusum au carré pour la période 1987-2010

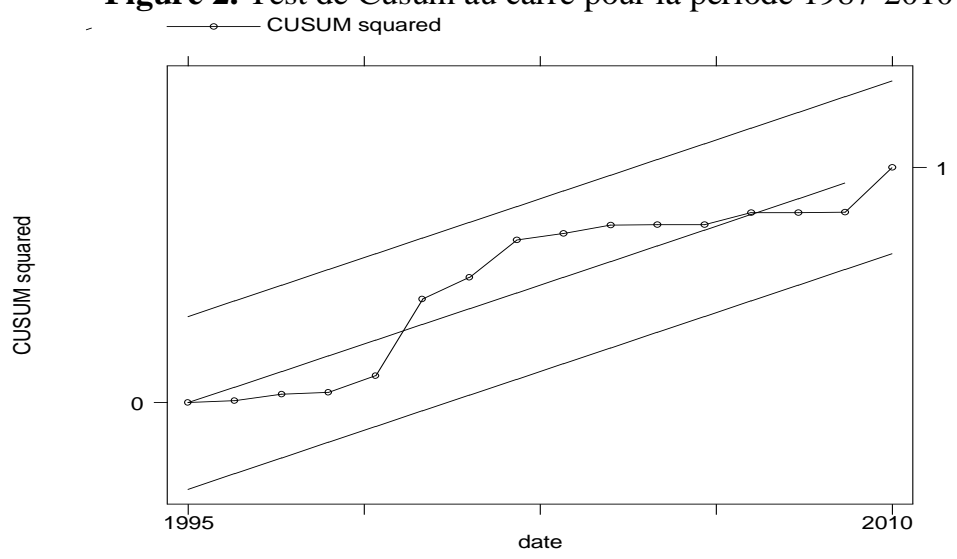

Source : l'auteur

Les signes positifs des coefficients associés au travail et au capital indiquent que ces facteurs de production favorisent la croissance en Côte d'Ivoire. En effet, une variation d'un pourcent du nombre d'emplois et du stock de capital induit une hausse respective de 2,10\% et 0,59\% du PIB réel de la Côte d'Ivoire. Par ailleurs, le signe négatif des coefficients associés aux indicateurs de diversification met en évidence un effet positif des efforts de diversification des exportations sur la croissance économique. Ce résultat est conforme à ceux de Herzer et Nowak-Lehnmann (2006) obtenus à partir de séries temporelles pour le Chili et ceux de Anwesha et Acharyya (2013) pour un panel 65 pays.

Enfin, la colonne (4) du Tableau 3 présente les résultats des estimations avec une décomposition de l'indice de Theil à la marge extensive et à la marge intensive. On observe que la diversification à la marge extensive n'a pas 
d'impact significatif en Côte d'Ivoire sur la période étudiée. Autrement dit, la diversification en direction des produits nouveaux n'aura pas eu d'impact significatif en Côte d'Ivoire. Ce sont plutôt les efforts de diversification des secteurs traditionnels qui a stimulé la production des richesses dans l'économie ivoirienne. Il s'agit par exemple des efforts de transformation de certains produits notamment les produits agricoles qui constituent le pilier de l'économie ivoirienne.

Ce résultat confirme l'observation de Cottet et al. (2012) concernant la pertinence de l'effet de la marge extensive sur la croissance. En effet, ces auteurs soulignent que l'apparition de nouveaux produits d'exportations ne pourrait être un moteur de croissance économique que si la marge extensive dominait la marge intensive. Malheureusement, cela semble ne pas être le cas en Côte d'Ivoire. Par ailleurs, il y a très peu de produits qui résulte d'un processus de remontée de la chaîne de la valeur ajoutée ou d'un processus d'innovation ou d'une simple imitation.

\section{Conclusion}

À la question de savoir si la diversification des exportations joue un rôle significatif dans le processus de croissance économique en Côte d'Ivoire, cette étude a entrepris l'estimation d'une équation de croissance par la méthode des moindres carrés généralisés. Les problèmes économétriques d'autocorrélation et d'hétéroscédasticité rencontrés ont été corrigés.

Et les résultats montrent que la diversification, surtout celle qui s'opère dans les secteurs traditionnels, contribue au processus de croissance économique sur la période 1987-2010. Cependant, sur la période 1962-1986, elle n'a pas eu d'impact significatif. Ces résultats invitent à une recommandation spécifique. La Côte d'Ivoire devrait renforcer son processus de diversification en se concentrant sur le développement de chaines des valeurs. Cela implique une réorganisation des secteurs traditionnelles d'exportations avant d'engager un processus de transformation de ces produits exportés vers une gamme plus importante, potentielle source de valeurs ajoutées. Une politique de remontée de filière avec des mécanismes de limitation des chocs sectoriels serait donc idéale.

\section{References:}

1. Acemoglu, D. \& Zilibotti, F. (1997). Was Prometheus Unbound by Chance? Risk, Diversification, and Growth. Journal of Political Economy 105(4), 709-751.

2. Anwesha, A. \& Acharyya, R. (2013). Export diversification, composition, and economic growth: Evidence from cross-country analysis. The Journal of International Trade \& Economic 
Development , An International and Comparative Review, 22 (7), 959992.

3. Arrow, K. J. (1962). The economic implications of learning by doing. The Review of Economic Studies, 29 (3), 155-173.

4. Banque Mondiale (2015). Performances économiques ivoiriennes depuis la fin de la crise post-électorale. Rapport sur la Situation Economique Récente en Côte d'ivoire. Consulté sur

5. http://documents.banquemondiale.org/curated/fr/1329814682301236 62/pdf/956010FRENCH0W0te0March020150Final0.pdf.

6. Banque Mondiale (2017). Les défis des compétences: pourquoi la Côte d'ivoire doit-elle reformer son système éducatif? Rapport sur la situation économique en Côte d'ivoire, Banque Mondiale. Quatrième Édition, consulté sur http://documents.banquemondiale.org/curated/fr/4896014852657574 00/pdf/112243-WP-FRENCH-PUBLIC-Cote-dIvoire-4th-economicupdate-feb2017-ligth.pdf.

7. Ben Hammouda, H., Oulmane, N. \& Sadni-Jallab, M. (2009). d'une diversification spontanée à une diversification organisée Quelles politiques pour diversifier les économies d'Afrique du Nord? Revue économique, 60(1), 133-155.

8. Cadot, O., Carrère, C. \& Strauss-Kahn, V. (2011). Export diversification: what's behind the hump? The Review of Economics and Statistics (MIT Press) 93(2), 590-605.

9. Chatri, A., Moussir, C.E. \& Bourdane, Y. (2018). Ouverture et vulnérabilité économique : cas des pays en développement. Revue "Repères et $P$ erspectives Economiques", consulté sur

10. https://www.researchgate.net/deref/https\%3A\%2F\%2Frevues.imist.m a\%2Findex.php\%3Fjournal\%3Drpe\%26page\%3Darticle\%26op\%3D view\%26path\%255B\%255D\%3D14784.

11. Cottet, C., Madariaga, N. \& Jégou, N. (2012). La diversification des exportations en zone franc: degré, sophistication et dynamique. Macroéconomie \& Développement (3).

12. Ehrhart, H. (2015). Les enjeux de la nouvelle croissance ivoirienne. Macroéconomie \& Développement, (20).

13. Fonds Monétaire International (2014). Assurer la croissance à long terme et la stabilité macroéconomique dans les pays à faible revenu: rôle de la transformation structurelle et de la diversification. Document de politique générale.

14. Greenaway, D., Morgan, W. \& Wright, P. (1999). Exports, export composition and growth. The Journal of International Trade \& Economic Development , 8 (1), 41-51. 
15. Herzer, D. \& Nowak-Lehnmann, F. (2006). What does export diversification do for growth? An econometric analysis. Applied Economics, volume 38, 15, Pages 1825-1838.

16. Hummels, D. \& Klenow, P. J. (2005). The Variety and Quality of a Nation's Exports. American Economic Review, 95(3), 704-723.

17. Keho, Y. (2012). Le rôle des facteurs institutionnels dans le développement financier et économique des pays de l'UEMOA. Revue économique et monétaire (12).

18. Klinger, B. \& Lederman, D. (2011). Export discoveries, diversification and barriers to entry. Economic Systems 35(1), 64-83.

19. Melitz, M. J. (2003). The Impact of Trade on Intra-Industry Reallocations and Aggregate Industry Productivity. Econometrica, 71(6), 1695-1725.

20. Prebisch, R. (1959). Commercial Policy in Under-Developed Countries. American Economic Review (American Economic Association) 49(2), 251-273.

21. Romer, P. (1986). Increasing Return and Long-Run Growth. Journal of Political Economy, 94(5), 1002-1037.

22. Romer, P. (1990). Endogenous Technological Change. Journal of Political Economy, 98, 71-102.

23. Solow, R. (1957). Technical change and the aggregate production function. Review of Economics and Statistics, 39, 312-320. 